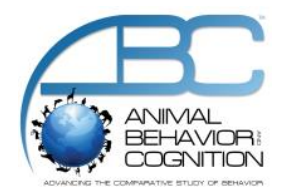

\title{
Mother-Offspring Signature Whistle Similarity and Patterns of Association in Atlantic Spotted Dolphins (Stenella frontalis)
}

\author{
Sara E. Bebus ${ }^{1,2}$ and Denise L. Herzing ${ }^{1,3}$
}

\author{
${ }^{1}$ The Wild Dolphin Project \\ ${ }^{2}$ University of Memphis \\ ${ }^{3}$ Florida Atlantic University \\ *Corresponding author (Email: sarabebus@gmail.com)
}

Citation - Bebus, S. E., \& Herzing, D. L. (2015). Mother-offspring signature whistle similarity and patterns of association in Atlantic spotted dolphins (Stenella frontalis). Animal Behavior and Cognition, 2(1), 71-87. doi: 10.12966/abc.02.06.2015

\begin{abstract}
We assessed the degree of similarity between signature whistles of mother and offspring pairs in freeliving Atlantic spotted dolphins (Stenella frontalis). As a means of qualitative evaluation, judges compared the timefrequency contour patterns of signature whistle spectrograms. We also evaluated quantitative measurements of whistle frequency and duration. All five female offspring produced signature whistles that were similar to their mothers' whistles by a least one means of comparison, whereas two of the four male offspring produced signature whistles that were dissimilar from their mothers' by both methods of comparison. However, statistically, male offspring were just as likely to produce signature whistles like their mothers' as female offspring $(p=0.167)$. We compared whistle similarity between mothers and offspring to the degree of association for each pair. Offspring that most often associated with their mothers when they were four years of age had whistles that were more similar to their mothers' whistles both qualitatively $(r(4)=0.92, p=0.009)$ and quantitatively $(r(4)=0.92, p=0.004)$. Because signature whistles are developed within a dolphin's first year, our finding likely reflects the level of bond between mother and calf.
\end{abstract}

Keywords - Signature whistle, Atlantic spotted dolphin, Stenella frontalis, Vocal communication, Vocal learning, Whistle ontogeny

Dolphins are long-lived social mammals with a sophisticated vocal communication system, which includes signature whistles, a type of stereotyped, frequency-modulated whistle that is unique to an individual. Caldwell and Caldwell (1965) first proposed the signature whistle hypothesis from the observation that captive bottlenose dolphins (Tursiops truncatus) produce individually distinctive "signature" whistles, which are emitted more often than any other whistle. Our knowledge of signature whistles is almost entirely based on bottlenose dolphin studies (Harley, 2008; Janik \& Sayigh, 2013; Tyack, 1997). However, signature whistles have been described for the Atlantic spotted dolphin (Stenella frontalis: Caldwell \& Caldwell, 1966; Caldwell, Caldwell, \& Miller, 1973; Herzing, 1996) and a few other dolphin species (common dolphin, Delphinus delphis: Caldwell \& Caldwell, 1968; Pacific whitesided dolphin, Lagenorhynchus obliquidens: Caldwell \& Caldwell, 1971; Indo-Pacific bottlenose dolphin, T. aduncus: Gridley et al., 2014, as well as probable signature whistles in Risso's dolphin, Grampus griseus: Caldwell, Caldwell, \& Miller, 1969; Guiana dolphin, Sotalia guianesis: de Figueiredo \& Simão, 2009; Pacific humpback dolphin, Sousa chinensis: Van Parijs \& Corkeron, 2001). Research assessing signature whistles in Atlantic spotted dolphins is limited (Burris, 2004; Herzing, 1996; Herzing, Deecke, 
\& Brunnick, 1999), as a result, works cited in this paper refer to bottlenose dolphins unless otherwise noted.

A dolphin's signature whistle retains a basic contour, but may vary in many elements (Caldwell \& Caldwell, 1965). However, most dolphins have "individually characteristic frequency ranges" (Caldwell, Caldwell, \& Tyack, 1990). Signature whistles are likely used as cohesion calls that broadcast the identity of the whistler (Caldwell \& Caldwell, 1965; Janik, Sayigh, \& Wells, 2006; Janik \& Slater, 1998; Quick \& Janik, 2012; Sayigh, Janik, \& Wells, 2005; Sayigh et al., 1998; Smolker, Mann, \& Smuts, 1993). Signature whistles may be copied by conspecifics (Janik, 2000; King, Sayigh, Wells, Fellner, \& Janik, 2013), possibly as a label to address an individual (King \& Janik, 2013).

According to Caldwell and Caldwell (1979), individuals produce a signature whistle by the time they reach four to six months of age. The signature whistle a dolphin produces appears to be influenced by the calf's acoustic environment (Caldwell \& Caldwell, 1979; Fripp et al., 2005; Janik \& Slater, 1997; Miksis, Tyack, \& Buck, 2002; Sayigh, Tyack, Wells, \& Scott, 1990; Sayigh, Tyack, Wells, Scott, \& Irvine, 1995). For example, Miksis et al. (2002) suggest that captive-born dolphins may incorporate elements of human-made sounds (i.e., a trainer's whistle) into their signature whistles. Likewise, a calf's signature whistles may be influenced by prenatal exposure to their mother's whistle (Tyack \& Sayigh, 1997) or by the signature whistles of other dolphins in their environment (Fripp et al., 2005; Sayigh et al., 1990).

The signature whistles of male bottlenose dolphins more closely resemble the signature whistles of their mothers, whereas female offspring more often have signature whistles "not similar" to the signature whistles of their mothers (Sayigh et al., 1990, 1995). Signature whistles of Atlantic spotted dolphin mothers are most similar to their calves, as compared to the rest of the population (Herzing et al., 1999).

In the Caldwells' studies of bottlenose dolphins, signature whistles made up a larger percentage of overall whistle production in female calves than male calves, though there was no difference between sexes in the structure of the whistles (Caldwell \& Caldwell, 1979; Caldwell et al., 1990). Signature whistles are produced often during separations and reunions between mothers and calves (bottlenose: Smolker et al., 1993; Atlantic spotted: Herzing, 1996). Adult females rely on signature whistles for coordination of movements with their offspring and this importance may be reflected in the high production rates of juvenile female dolphins.

The signature whistles of female bottlenose and Atlantic spotted dolphins remain stable over periods of many years (bottlenose, 12 years: Sayigh et al., 1990; spotted, between age classes: Burris, 2004). Stable signature whistles may be advantageous for female dolphins in maintaining communication with offspring and long-term relationships with other females (spotted: Herzing \& Brunnick, 1997). Signature whistles of male dolphins are less stable than those of females. Males within a coalition may form an "alliance signature," as it may be more important for highly associated males to be identified as a member of a tightly bonded pair or group rather than as an individual (Smolker \& Pepper, 1999). Sexspecific differences in stability and use of signature whistles are likely to be reflected in the development of signature whistles in calves.

\section{Present Study}

Bottlenose and Atlantic spotted dolphins use signature whistles in the same manner (i.e., reunions and separations, particularly between mothers and calves; spotted: Herzing, 1996; bottlenose: Janik \& Slater, 1998; Smolker et al., 1993). However, the two species have different life histories, such as dispersal patterns and foraging strategies (spotted: Herzing \& Johnson, 1997; bottlenose: Wells \& Scott, 1999). We investigated whether the signature whistles of male Atlantic spotted dolphin calves are more likely to be similar to the signature whistles of their mothers, as previous research has found with bottlenose dolphins (Sayigh et al., 1990, 1995). We also compared signature whistle similarity of mothercalf dyads with the degree of association between the pair for the calf's first four years. 


\section{Method}

\section{Study Population}

Whistles were recorded from a population of free-living Atlantic spotted dolphins off Grand Bahama Island in the Bahamas. This population is the focus of a long-term research study, which started in 1985 (Herzing 1996, 1997). Individual dolphins in the population have been identified by variations in dorsal fins, flukes, and constellations of spots, and sex has been determined by observation of the genital region (Herzing, 1997). The dolphins in this area of the Bahamas have been habituated to human swimmers since the mid-1970s. The shallow sand bank and high water visibility allow for underwater access to dolphin behavior.

\section{Recorded Whistles}

Whistles were extracted from underwater video and sound recordings made between 1987 and 2005 with video cameras (Sony CCDV9 8mm, Yaschica KV 1 Hi 8mm) and a Labcore 76 hydrophone, flat to $22 \mathrm{kHz}$ with a $-192 \mathrm{~dB}$ re $1 \mu \mathrm{Pa}$. Whistles were assigned to specific individuals when a dolphin was alone in the camera/hydrophone vicinity, in sole proximity $(<1 \mathrm{~m})$ of the camera/hydrophone, or showed simultaneous bubble emissions correlated with a whistle. Although amplitude can be variable in dolphin vocalizations, individuals more than $5 \mathrm{~m}$ away from the recording equipment never emitted whistles as loud as dolphins within $1 \mathrm{~m}$. If a group of dolphins was present, intensity of the whistle was only used to determine the whistling individual when one dolphin was close to the hydrophone $(<1 \mathrm{~m})$ and the rest of the group was further away $(\geq 5 \mathrm{~m})$. Some previous studies have relied on only bubble streams to identify the vocalizing individuals (e.g., McCowan \& Reiss, 1995a,b,; Reiss, 1988; see also Fripp, 2005). However, our unique underwater viewing allowed us to note not only bubble streams, but also the directional orientation and proximity of individuals to the recording equipment and the lack of other dolphins in the immediate area.

Whistles were digitized from audio recordings using Raven Pro 1.1 software (Cornell University, Ithaca, NY, USA) at $44.1 \mathrm{kHz}$ sampling rate. All spectrograms were made with Raven Pro 1.1 with a Hann window, 512 samples, and time grid spacing of 256 samples $(5.80 \mathrm{mS})$.

\section{Whistle Sample}

Mother-offspring pairs with a minimum of ten recorded signature whistles per individual were included (a minimum used in other studies, spotted: Burris, 2004; bottlenose: Sayigh et al., 1990). Nine mother-offspring pairs comprised from 15 individuals met this criterion. The pairs included six different females: four females with a single offspring, one with two offspring, and one with three offspring.

Most whistles have repeated elements, termed 'loops.' The number of loops in a signature whistle is generally consistent for an individual, and the initial or terminal loop may be distinct in frequency from the other loops (Sayigh et al., 1990). We measured multi-loop whistles as a single unit. Each whistle was defined by either continuous, unbroken loops, or loops that do not have any breaks greater than 0.1 second. Although some researchers have used single loops as a unit of measurement, we choose multiloops because individuals consistently produced the same number of loops in their signature whistles (see also Esch, Sayigh, \& Wells, 2009). We utilized two different methods to compare signature whistles between individuals: a qualitative measurement of whistle contour similarity that has been used previously in whistle comparison studies (Sayigh et al., 1990, 1995; Watwood, Tyack, \& Wells, 2004) and a quantitative method to examine the basic measures of whistles (e.g., frequency and duration). 


\section{Qualitative: Judge Comparison of Whistle Contour}

Two of the clearest spectrograms were chosen as representative signature whistles from each individual for a total of 30 whistles. Because signature whistles in Atlantic spotted dolphins are stereotyped and stable over time, we did not feel that it was necessary to include more than two whistles from each individual (Burris, 2004; Herzing, 1996).

Three judges rated the similarity of the whistle contours between mother-offspring and nonrelated pairs on a 1-to-5 scale (with 1 as "not similar," 2 as "slightly similar," 3 as "somewhat similar," 4 as "similar," and 5 as "very similar."). A score of 5 did not require that the whistles were identical. Judges were not given information about the identity of the dolphins, and each whistle was labeled with a random number. Three graduate students without any experience with signature whistles acted as judges.

The whistles of four additional dolphins were included in the sample (two whistles from each individual) to increase the number of comparisons between non-related dolphins. Each of the 38 whistles in the sample was compared with each of the others in a randomized order, which varied for each judge. Spectrograms were produced in Raven 1.1 with identical $\mathrm{x}$ - and y-axes and viewed by the judges on a computer monitor (examples in Figure 1). Each judge was presented with a series of seven practice pairs. Practice pairs were comprised of signature whistles from dolphins not used in this study and allowed the judges to become comfortable with comparing the spectrograms. The practice pairs were presented at the beginning, the half-way point, and the end of the judging sessions. Each judge was given the same practice set. Two of the judges also analyzed a subset of whistle pairs a second time. This additional analysis and the practice pairs were used to test intra-rater reliability. Inter- and intra-rater reliability were assessed with Cohen's $\kappa$ coefficients.

A mean score of similarity was calculated from all comparisons (three judges comparing two whistles per dolphin for a total of twelve similarity scores per dolphin pair). We compared each mother's similarity mean score with her offspring to: 1 ) her mean similarity score with all unrelated individuals in the study, 2) the 5-point scale given to the judges, and 3) the scale used by Sayigh et al. (1995) in which the 1 to 5 rating scale was simply divided into three equal categories (scores greater than 3.66 were defined as "similar," scores between 3.66 and 2.34 as "somewhat similar," and those below 2.34 as "not similar"). We used this final method in order to directly compare our results to the previous research in bottlenose dolphins, as the instructions given to the judges in our study were very similar to those given in Sayigh et al. (1995).

\section{Quantitative: Whistle Parameter Comparison}

While signature whistle contour is the key element of a whistle for individual recognition (Janik et al., 2006; Kershenbaum, Sayigh, \& Janik, 2013; Sayigh et al., 1998, 2005; Sayigh, Esch, Wells, \& Janik, 2007), we also compared additional parameters between related dolphins. Dolphins have been shown to produce whistles at "individually characteristic frequency ranges" (Caldwell et al., 1990), so we choose to examine quantitative whistles parameters in addition to contour similarity. For individuals with greater than ten recorded whistles, we chose ten signature whistles at random for measurements. This was to keep the sample size, and possible variance, consistent for each individual. For each whistle we measured: total duration, maximum and minimum frequencies, initial and terminal frequencies, change in frequency, number of loops, and duration of single loops. The means for each parameter were determined for each individual dolphin. Only the fundamental frequency was measured; any harmonics were ignored. All measurements were made using the measurement cursors in Raven 1.1. Values for each measured parameter were compared for each pair of related dolphins using a one-way analysis of variance (ANOVA). All statistical tests were run with SPSS 14.0 software (SPSS Inc., Chicago, IL, USA). 


\section{Sex-Based Differences}

To determine if signature whistle similarity between mothers and offspring differed based on offspring sex, we used a Fisher's exact test to compare the number of male and female calves with whistles similar to their mothers. For the Fisher's exact test, if the signature whistles of a pair were determined to be similar by either qualitative or quantitative means (as in Table 5), that pair was considered "similar." All other pairs were considered "not similar."
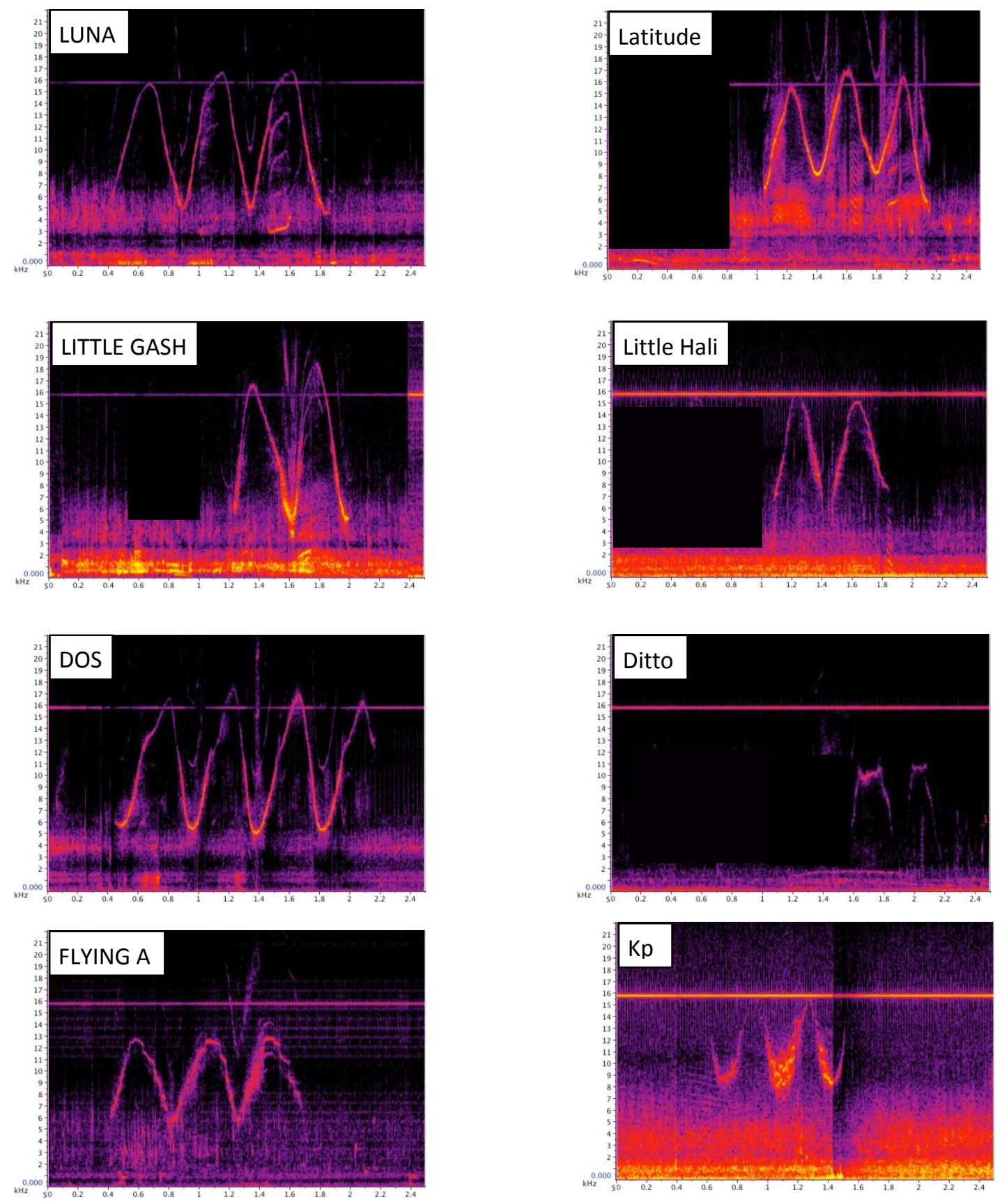

Figure 1. Representative spectrograms of the mother-offspring pairs determined to be most and least similar by judge scoring: Luna and male offspring Latitude (3.50), Little Gash and female offspring Little Hali (3.33), Dos and male offspring Ditto (1.67), and Flying A and female offspring KP (1.75). 


\section{Degree of Association}

Atlantic spotted dolphins live in fission-fusion societies, where the size and composition of a group changes over time, even within a single day (Welsh \& Herzing, 2008). Degree of association between two individuals in this type of fluctuating social environment can be quantified by a coefficient of association (COA). The COAs of this population were previously determined using the half-weight index (HWI) and SOCPROG modeling software (version 2.3) (Elliser \& Herzing, 2012, 2014; Welsh \& Herzing, 2008). Analysis with the HWI provided a value between 0 and 1 for each dyad, based on how often two individuals are seen together and how often they are seen separately (Cairns \& Schwager, 1987). Individuals were considered to be associating if they were observed in the same group (spotted: Elliser \& Herzing, 2012; Welsh \& Herzing, 2008).

The highest COAs are found between a mother and her calf. COA values for mother-infant pairs in this population of Atlantic spotted dolphins are typically between 0.85 and 0.96 (Herzing \& Brunnick, 1997), but individual variations in COAs occur between mother-calf pairs, with some nearly always observed together and other pairs often observed apart. Additionally, there was no sex difference in mother-calf COAs during the first year of life, when the signature whistle is developed (Herzing \& Brunnick, 1997). Measures of whistle similarity were compared to the COA of each mother-offspring pair. COAs for each year from birth to year 4 were analyzed separately with a Pearson's correlation.

\section{Results}

\section{Qualitative Similarity Ratings}

There was moderate to high intra-rater agreement of scores (Table 1). Inter-rater reliability was significant for all pair-wise comparisons between judges (Table 1).

We used three different scales to define the degree of similarity between mother and offspring pairs (Table 2). First, the similarity scores from each judge were averaged and compared between motheroffspring and non-relative pairs. Four mother-female offspring pairs (Little Gash-Little Hali, FlashlightFlare, Luna-Diamond, Gemini-Galaxy), and two mother-male offspring pairs (Luna-Latitude, DosLucaya) were more similar to each other than to non-relatives. Secondly, using the description given to the judges for the 1-to-5 scale of similarity, three pairs were "somewhat similar," four were "slightly similar," and two were "not similar" (Table 2). The highest score of similarity between individuals was between a mother and her male offspring (Luna-Latitude), with a score of 3.50 (Figure 1). The lowest score of similarity was 1.67 and was also between a mother and her male offspring (Dos-Ditto). Comparing mean similarity scores with non-relative pairs yielded results no different than those from using the 5-point scale. Lastly, we used the scale defined by Sayigh et al. (1995). All pairs previously considered "similar" by the first method discussed above were considered "somewhat similar," by the Sayigh et al. (1995) scale, and pairs considered "not similar" were unchanged (Table 2).

\section{Quantitative Measurements}

The numbers of loops per whistle ranged from 1 to 6 , total whistle duration from 0.17 to $2.70 \mathrm{~s}$, duration of individual loops from 0.076 to $0.65 \mathrm{~s}$, minimum frequency from 2.43 to $8.90 \mathrm{kHz}$, maximum frequency from 10.02 to $21.30 \mathrm{kHz}$, change in frequency from 1.36 to $19.34 \mathrm{kHz}$, initial frequency from 2.42 to $14.86 \mathrm{kHz}$, and terminal frequency from 2.55 to $16.34 \mathrm{kHz}$. Mean values for these whistle measurements from each individual dolphin are listed in Table 3. 
Table 1

Inter-rater and Intra-rater Reliability of Whistle Similarity Scoring by Judges

\begin{tabular}{|c|c|c|c|}
\hline & Judge A & Judge B & Judge C \\
\hline Judge A & $\begin{array}{c}\kappa=0.39 \\
\mathrm{p}=0.001 \\
\mathrm{n}=22\end{array}$ & $\begin{array}{c}\kappa=0.10 \\
\mathrm{p}<0.001 \\
\mathrm{n}=696\end{array}$ & $\begin{array}{c}\kappa=0.040 \\
\mathrm{p}=0.002 \\
\mathrm{n}=696\end{array}$ \\
\hline Judge B & & $\begin{array}{c}\kappa=0.41 \\
\mathrm{p}<0.001 \\
\mathrm{n}=200\end{array}$ & $\begin{array}{c}\kappa=0.28 \\
\mathrm{p}<0.001 \\
\mathrm{n}=703\end{array}$ \\
\hline Judge C & & & $\begin{array}{c}\kappa=1.000 \\
\mathrm{p}<0.001 \\
\mathrm{n}=7\end{array}$ \\
\hline
\end{tabular}

Table 2

Qualitative Results Presented with three Guidelines of Defining Similarity

\begin{tabular}{|c|c|c|c|c|c|c|}
\hline \multicolumn{2}{|c|}{ Dolphin Pairs } & \multirow{2}{*}{$\begin{array}{c}\text { Mean } \\
\text { Similarity } \\
\text { Score }\end{array}$} & \multirow{2}{*}{$\begin{array}{c}\text { Offspring Sex } \\
\mathrm{M}\end{array}$} & \multirow{2}{*}{$\begin{array}{c}\text { Compared to } \\
\text { Other Individuals } \\
\text { Similar }\end{array}$} & \multirow{2}{*}{$\begin{array}{c}\text { Judge Instructional } \\
\text { Scale } \\
\text { Somewhat Similar } \\
(3-4)\end{array}$} & \multirow{2}{*}{$\begin{array}{c}\text { As in } \\
\text { Sayigh et al. } \\
(1995) \\
\text { Somewhat Similar } \\
(2.34-3.66)\end{array}$} \\
\hline LUNA (2.49) & Latitude (2.35) & & & & & \\
\hline $\begin{array}{l}\text { LITTLE GASH } \\
(2.42)\end{array}$ & $\begin{array}{l}\text { Little Hali } \\
(2.62)\end{array}$ & 3.33 & $\mathrm{~F}$ & & & \\
\hline $\begin{array}{l}\text { FLASH- LIGHT } \\
(2.02)\end{array}$ & $\begin{array}{l}\text { Flare } \\
(1.87)\end{array}$ & 3.08 & $\mathrm{~F}$ & & & \\
\hline LUNA (2.49) & $\begin{array}{l}\text { Diamond } \\
(2.22)\end{array}$ & 2.83 & $\mathrm{~F}$ & & $\begin{array}{c}\text { Slightly Similar } \\
\qquad(2-<3)\end{array}$ & \\
\hline $\begin{array}{l}\text { DOS } \\
(2.42)\end{array}$ & Lucaya (2.45) & 2.83 & M & & & \\
\hline GEMINI (1.16) & Galaxy (1.39) & 2.42 & $\mathrm{~F}$ & & & \\
\hline $\begin{array}{l}\text { DOS } \\
(2.42)\end{array}$ & $\begin{array}{l}\text { Deni } \\
(2.42)\end{array}$ & 2.25 & $\mathrm{~F}$ & Not Similar & & $\begin{array}{l}\text { Not Similar } \\
(<2.34)\end{array}$ \\
\hline $\begin{array}{l}\text { FLYING A } \\
(2.31)\end{array}$ & $\begin{array}{l}\mathrm{Kp} \\
(2.10)\end{array}$ & 1.75 & M & & $\begin{array}{c}\text { Not Similar } \\
\quad(<2)\end{array}$ & \\
\hline $\begin{array}{l}\text { DOS } \\
(2.42)\end{array}$ & $\begin{array}{l}\text { Ditto } \\
(1.76)\end{array}$ & 1.67 & M & $\downarrow$ & $\downarrow$ & $\downarrow$ \\
\hline
\end{tabular}

Note. Names of mothers are in all capital letters and offspring names are in lowercase. Judges scored whistle similarity on a 1-to5 scale. Mean similarity score with all non-related dolphins in this study is in parentheses below the individual's name. 
Table 3

Means, Standard Deviations (SD), and Sample Sizes for Each Individual Dolphin

\begin{tabular}{|c|c|c|c|c|c|c|c|c|c|}
\hline Dolphin Name & & $\begin{array}{l}\text { Number of } \\
\text { Loops }\end{array}$ & $\begin{array}{c}\text { Total } \\
\text { Duration } \\
\text { (sec) }\end{array}$ & $\begin{array}{l}\text { Single Loop } \\
\text { Duration } \\
(\mathrm{sec})\end{array}$ & $\begin{array}{l}\text { Minimum } \\
\text { Frequency } \\
(\mathrm{kHz})\end{array}$ & $\begin{array}{c}\text { Maximum } \\
\text { Frequency }(\mathrm{kHz})\end{array}$ & $\begin{array}{c}\text { Change in } \\
\text { Frequency }(\mathrm{kHz})\end{array}$ & $\begin{array}{c}\text { Initial } \\
\text { Frequency }(\mathrm{kHz})\end{array}$ & $\begin{array}{c}\text { Terminal } \\
\text { Frequency }(\mathrm{kHz})\end{array}$ \\
\hline \multirow[t]{3}{*}{ DOS } & Mean & 2.41 & 1.15 & 0.43 & 4.67 & 17.40 & 12.77 & 5.87 & 11.55 \\
\hline & $\mathrm{SD}$ & 0.87 & 0.39 & 0.06 & 0.48 & 1.48 & 1.33 & 1.98 & 2.25 \\
\hline & $\mathrm{n}$ & 17 & 17 & 41 & 17 & 14 & 14 & 17 & 17 \\
\hline \multirow[t]{3}{*}{ Deni } & Mean & 2.58 & 1.05 & 0.41 & 4.60 & 11.61 & 7.01 & 5.23 & 5.38 \\
\hline & $\mathrm{SD}$ & 0.74 & 0.29 & 0.05 & 0.80 & 1.68 & 1.33 & 1.10 & 1.09 \\
\hline & $\mathrm{n}$ & 55 & 55 & 142 & 55 & 55 & 55 & 55 & 55 \\
\hline \multirow{3}{*}{ Ditto } & Mean & 1.33 & 0.32 & 0.22 & 6.20 & 10.88 & 4.69 & 7.89 & 6.39 \\
\hline & SD & 0.49 & 0.20 & 0.06 & 1.35 & 0.56 & 1.48 & 1.31 & 1.69 \\
\hline & $\mathrm{n}$ & 12 & 12 & 16 & 12 & 12 & 12 & 12 & 12 \\
\hline \multirow[t]{3}{*}{ Lucaya } & Mean & 2.25 & 0.86 & 0.37 & 5.83 & 17.18 & 11.39 & 7.24 & 8.23 \\
\hline & $\mathrm{SD}$ & 0.69 & 0.28 & 0.066 & 1.34 & 1.27 & 1.82 & 1.55 & 2.54 \\
\hline & $\mathrm{n}$ & 36 & 36 & 81 & 35 & 34 & 33 & 36 & 36 \\
\hline FLASH- & Mean & 2.70 & 1.22 & 0.38 & 7.67 & 14.12 & 6.51 & 10.95 & 11.02 \\
\hline \multirow{2}{*}{ LIGHT } & $\mathrm{SD}$ & 1.06 & 0.37 & 0.067 & 0.55 & 0.99 & 1.03 & 2.47 & 2.13 \\
\hline & $\mathrm{n}$ & 10 & 10 & 27 & 9 & 7 & 7 & 10 & 10 \\
\hline \multirow[t]{3}{*}{ Flare } & Mean & 2.69 & 1.08 & 0.35 & 7.21 & 15.52 & 8.50 & 10.04 & 11.99 \\
\hline & SD & 1.32 & 0.44 & 0.049 & 0.65 & 1.52 & 1.60 & 1.60 & 2.08 \\
\hline & $\mathrm{n}$ & 13 & 13 & 35 & 13 & 8 & 8 & 13 & 13 \\
\hline FLYING & Mean & 3.56 & 1.40 & 0.39 & 4.25 & 13.92 & 9.67 & 5.62 & 5.97 \\
\hline \multirow[t]{2}{*}{ A } & $\mathrm{SD}$ & 1.04 & 0.42 & 0.037 & 0.99 & 0.78 & 1.37 & 1.56 & 1.24 \\
\hline & $\mathrm{n}$ & 18 & 18 & 64 & 18 & 18 & 18 & 18 & 18 \\
\hline \multirow[t]{3}{*}{$\mathrm{Kp}$} & Mean & 1.93 & 0.75 & 0.34 & 7.89 & 15.27 & 7.35 & 10.36 & 12.56 \\
\hline & $\mathrm{SD}$ & 0.62 & 0.25 & 0.062 & 0.61 & 1.48 & 1.86 & 2.15 & 2.05 \\
\hline & $\mathrm{n}$ & 14 & 14 & 27 & 14 & 12 & 12 & 14 & 14 \\
\hline \multirow[t]{3}{*}{ GEMINI } & Mean & 3.25 & 1.15 & 0.35 & 3.88 & 11.72 & 8.49 & 10.54 & 4.86 \\
\hline & $\mathrm{SD}$ & 0.75 & 0.34 & 0.14 & 0.90 & 0.22 & 1.65 & 0.40 & 1.11 \\
\hline & $\mathrm{n}$ & 12 & 12 & 39 & 10 & 12 & 12 & 12 & 12 \\
\hline \multirow[t]{3}{*}{ Galaxy } & Mean & 3.00 & 1.12 & 0.31 & 7.23 & 14.47 & 7.21 & 12.55 & 7.87 \\
\hline & $\mathrm{SD}$ & 0.78 & 0.34 & 0.12 & 0.53 & 1.14 & 1.24 & 0.91 & 1.40 \\
\hline & $\mathrm{n}$ & 21 & 21 & 63 & 21 & 19 & 19 & 21 & 21 \\
\hline LITTLE & Mean & 2.58 & 1.04 & 0.40 & 4.87 & 17.20 & 12.39 & 6.35 & 5.41 \\
\hline \multirow[t]{2}{*}{ GASH } & SD & 0.79 & 0.36 & 0.080 & 0.80 & 2.22 & 2.69 & 1.85 & 1.12 \\
\hline & $\mathrm{n}$ & 12 & 12 & 31 & 12 & 10 & 10 & 12 & 12 \\
\hline \multirow[t]{3}{*}{ Little Hali } & Mean & 2.48 & 1.10 & 0.42 & 5.73 & 16.44 & 10.81 & 7.85 & 6.27 \\
\hline & $\mathrm{SD}$ & 0.73 & 0.35 & 0.079 & 1.31 & 2.93 & 2.10 & 1.83 & 1.37 \\
\hline & $\mathrm{n}$ & 23 & 23 & 57 & 23 & 20 & 20 & 23 & 23 \\
\hline
\end{tabular}


Bebus \& Herzing 79

Table 3 (cont.)

\begin{tabular}{|c|c|c|c|c|c|c|c|c|c|}
\hline \multirow[t]{3}{*}{ LUNA } & Mean & 2.74 & 1.30 & 0.47 & 4.14 & 17.77 & 13.63 & 4.55 & 4.84 \\
\hline & SD & 0.85 & 0.41 & 0.047 & 0.59 & 1.15 & 1.17 & 0.80 & 0.86 \\
\hline & $\mathrm{n}$ & 66 & 66 & 181 & 66 & 61 & 61 & 66 & 66 \\
\hline \multirow[t]{3}{*}{ Diamond } & Mean & 2.73 & 1.17 & 0.42 & 6.39 & 18.77 & 12.27 & 10.03 & 6.87 \\
\hline & SD & 0.47 & 0.22 & 0.03 & 1.27 & 1.45 & 1.72 & 2.41 & 2.36 \\
\hline & $\mathrm{n}$ & 11 & 11 & 30 & 11 & 7 & 7 & 11 & 11 \\
\hline \multirow[t]{3}{*}{ Latitude } & Mean & 3.51 & 1.32 & 0.37 & 6.09 & 17.39 & 11.26 & 7.99 & 6.57 \\
\hline & SD & 0.92 & 0.38 & 0.048 & 1.14 & 1.10 & 1.53 & 1.58 & 1.63 \\
\hline & $\mathrm{n}$ & 49 & 48 & 172 & 49 & 46 & 46 & 49 & 49 \\
\hline \multirow[t]{3}{*}{ Average } & Mean & 2.65 & 1.07 & 0.38 & 5.78 & 15.31 & 9.60 & 8.20 & 7.72 \\
\hline & SD & 0.81 & 0.34 & 0.066 & 0.89 & 1.33 & 1.59 & 1.57 & 1.66 \\
\hline & $\mathrm{n}$ & 24.60 & 24.53 & 67.07 & 24.33 & 22.33 & 22.27 & 24.60 & 24.60 \\
\hline Totals & & 369 & 368 & 1006 & 365 & 335 & 334 & 369 & 369 \\
\hline
\end{tabular}

Note. In some cases, maximum frequency could not be determined due to attenuation of the signal at the highest frequencies. For this reason, the sample size is not consistent across measures for some individuals. Names of mothers are in all capital letters. Offspring names are in lowercase listed below their mother. 
Table 4

ANOVA Results between Mother and Offspring Pairs for Measured Parameters

\begin{tabular}{|c|c|c|c|c|c|c|c|c|c|}
\hline Mother & Offspring (sex) & $\begin{array}{c}\text { Number of } \\
\text { Loops }\end{array}$ & $\begin{array}{c}\text { Total } \\
\text { Duration }\end{array}$ & $\begin{array}{l}\text { Minimum } \\
\text { Frequency }\end{array}$ & $\begin{array}{l}\text { Maximum } \\
\text { Frequency }\end{array}$ & $\begin{array}{l}\text { Change in } \\
\text { Frequency }\end{array}$ & $\begin{array}{c}\text { Initial } \\
\text { Frequency }\end{array}$ & $\begin{array}{l}\text { Terminal } \\
\text { Frequency }\end{array}$ & $\begin{array}{c}\text { \# of Similar } \\
\text { Parameters }\end{array}$ \\
\hline \multirow{3}{*}{ DOS } & Lucaya (M) & $\begin{array}{l}F=13.50 \\
p=0.002\end{array}$ & $\begin{array}{c}38.30 \\
<0.001\end{array}$ & $\begin{array}{l}17.08 \\
0.001\end{array}$ & $\begin{array}{l}1.04 \\
0.32\end{array}$ & $\begin{array}{l}10.57 \\
0.004\end{array}$ & $\begin{array}{l}2.82 \\
0.11\end{array}$ & $\begin{array}{c}3.64 \\
0.073\end{array}$ & 3 \\
\hline & Ditto (M) & $\begin{array}{l}F=33.80 \\
p<0.001\end{array}$ & $\begin{array}{l}163.28 \\
<0.001\end{array}$ & $\begin{array}{c}43.75 \\
<0.001\end{array}$ & $\begin{array}{c}142.22 \\
<0.001\end{array}$ & $\begin{array}{l}266.42 \\
<0.001\end{array}$ & $\begin{array}{c}8.23 \\
0.010\end{array}$ & $\begin{array}{c}31.13 \\
<0.001\end{array}$ & 0 \\
\hline & Deni $(F)$ & $\begin{array}{l}\mathrm{F}=0.00 \\
\mathrm{p}=1.00\end{array}$ & $\begin{array}{l}1.77 \\
0.20\end{array}$ & $\begin{array}{l}1.50 \\
0.24\end{array}$ & $\begin{array}{c}45.93 \\
<0.001\end{array}$ & $\begin{array}{c}54.19 \\
<0.001\end{array}$ & $\begin{array}{l}0.99 \\
0.33\end{array}$ & $\begin{array}{c}59.37 \\
<0.001\end{array}$ & 4 \\
\hline $\begin{array}{c}\text { FLASH- } \\
\text { LIGHT }\end{array}$ & Flare $(\mathrm{F})$ & $\begin{array}{l}F=0.00 \\
p=1.00\end{array}$ & $\begin{array}{l}0.68 \\
0.42\end{array}$ & $\begin{array}{c}4.22 \\
0.056\end{array}$ & $\begin{array}{c}4.28 \\
0.059\end{array}$ & $\begin{array}{c}7.97 \\
0.014\end{array}$ & $\begin{array}{l}1.95 \\
0.18\end{array}$ & $\begin{array}{l}0.91 \\
0.35\end{array}$ & 6 \\
\hline FLYING A & $\mathrm{Kp}(\mathrm{M})$ & $\begin{array}{l}F=27.74 \\
p<0.001\end{array}$ & $\begin{array}{c}29.53 \\
<0.001\end{array}$ & $\begin{array}{l}110.21 \\
<0.001\end{array}$ & 10.450 .005 & $\begin{array}{c}3.61 \\
0.074\end{array}$ & $\begin{array}{c}25.09 \\
<0.001\end{array}$ & $\begin{array}{c}77.14 \\
<0.001\end{array}$ & 1 \\
\hline GEMINI & Galaxy (F) & $\begin{array}{l}F=2.06 \\
p=0.17\end{array}$ & $\begin{array}{l}0.26 \\
0.62\end{array}$ & $\begin{array}{l}107.53 \\
<0.001\end{array}$ & $\begin{array}{l}145.65 \\
<0.001\end{array}$ & $\begin{array}{c}7.47 \\
0.014\end{array}$ & $\begin{array}{c}63.87 \\
<0.001\end{array}$ & $\begin{array}{c}35.52 \\
<0.001\end{array}$ & 2 \\
\hline $\begin{array}{l}\text { LITTLE } \\
\text { GASH }\end{array}$ & Little Hali (F) & $\begin{array}{l}F=0.11 \\
p=0.75\end{array}$ & $\begin{array}{l}0.20 \\
0.66\end{array}$ & $\begin{array}{l}0.48 \\
0.50\end{array}$ & $\begin{array}{l}2.70 \\
0.12\end{array}$ & $\begin{array}{c}3.36 \\
0.083\end{array}$ & $\begin{array}{l}0.71 \\
0.41\end{array}$ & $\begin{array}{l}1.64 \\
0.22\end{array}$ & 7 \\
\hline \multirow[t]{2}{*}{ LUNA } & Diamond $(F)$ & $\begin{array}{l}F=0.00 \\
p=1.00\end{array}$ & $\begin{array}{l}1.15 \\
0.30\end{array}$ & $\begin{array}{c}24.62 \\
<0.001\end{array}$ & $\begin{array}{c}4.34 \\
0.055\end{array}$ & $\begin{array}{l}2.54 \\
0.13\end{array}$ & $\begin{aligned} & 39.25 \\
< & 0.001\end{aligned}$ & $\begin{array}{c}4.79 \\
0.042\end{array}$ & 4 \\
\hline & Latitude (M) & $\begin{array}{l}F=12.52 \\
p=0.002\end{array}$ & $\begin{array}{c}0.186 \\
0.67\end{array}$ & $\begin{array}{c}34.28 \\
<0.001\end{array}$ & $\begin{array}{l}1.58 \\
0.23\end{array}$ & $\begin{array}{c}26.86 \\
<0.001\end{array}$ & $\begin{array}{c}27.35 \\
<0.001\end{array}$ & $\begin{array}{c}7.69 \\
0.013\end{array}$ & 2 \\
\hline
\end{tabular}

Note. $n=10$ whistles for each individual, significant differences are bolded $(p<0.05)$ 


\section{Quantitative Similarity Comparisons}

Only one pair of related dolphins (Little Gash and her female offspring Little Hali) was similar for all measured parameters (Table 4). Flashlight and her female offspring, Flare, were the only pair with just one parameter (change in frequency) that was significantly different. Dos and her male offspring, Ditto, differed for all parameters.

\section{Similarity Summary}

All five mother-female offspring pairs had similar signature whistles by the qualitative and/or quantitative method (Table 5). Two of the four mother-male offspring pairs had similar whistles by the qualitative and/or quantitative method. The other two mother-male offspring pairs were not determined to be similar by either method. However, likely due to small sample size, statistically, neither sex was more likely to produce signature whistles similar to their mothers $(p=0.167)$.

Table 5

Similarity Summary Table by Qualitative (contour similarity by judge scoring) and Quantitative Methods (number of similar parameters)

\begin{tabular}{llcc}
\hline & Pairs & Qualitative & Quantitative \\
\hline Mother & Male & & \\
DOS & Lucaya & $\mathrm{S}$ & $\mathrm{S}$ \\
DOS & Ditto & $\mathrm{N}$ & $\mathrm{N}$ \\
FLYING A & Kp & $\mathrm{N}$ & $\mathrm{N}$ \\
LUNA & Latitude & $\mathrm{S}$ & $\mathrm{N}$ \\
& & & \\
Mother & Female & & \\
DOS & Deni & $\mathrm{N}$ & $\mathrm{S}$ \\
FLASHLIGHT & Flare & $\mathrm{S}$ & $\mathrm{S}$ \\
GEMINI & Galaxy & $\mathrm{S}$ & $\mathrm{N}$ \\
LITTLE GASH & Little Hali & $\mathrm{S}$ & $\mathrm{S}$ \\
LUNA & Diamond & $\mathrm{S}$ & $\mathrm{N}$ \\
\hline
\end{tabular}

Note. Each pair and method is listed as similar $(\mathrm{S})$ or not similar $(\mathrm{N})$. If at least three out of five whistle parameters (total duration, minimum, maximum, initial, and terminal frequency) were not significantly different for a pair, the whistles of the pair are determined to be similar. Pairs determined similar by either of the methods have been bolded.

\section{Degree of Association}

Whistle similarity was compared to COA for each mother-calf pair from birth through year 4 (Table 6). A positive correlation was found between COAs of offspring at 4 years of age and qualitative judge scoring of whistle similarity $(r(4)=0.92, p=0.009$, Figure 2a). Additionally, we found a positive correlation between COA in year 4 and the number of whistle parameters that were similar for mother and offspring pairs $(r(4)=0.92, p=0.004$, Figure $2 b)$. 
Table 6

Correlations Between COAs and Whistle Similarity in Mother-Offspring Pairs by Age of the Calf

\begin{tabular}{lcccc}
\hline & Year 1 & Year 2 & Year 3 & Year 4 \\
\hline Judge Score of Whistle Similarity & $\mathrm{r}(7)=-0.41$ & $\mathrm{r}(6)=0.022$ & $\mathrm{r}(5)=0.71$ & $\mathrm{r}(4)=0.92$ \\
& $\mathrm{p}=0.28$ & $\mathrm{p}=0.96$ & $\mathrm{p}=0.075$ & $\mathrm{p}=0.009$ \\
& & & & \\
Number of Similar Whistle Parameters & $\mathrm{r}(7)=-0.65$ & $\mathrm{r}(6)=0.33$ & $\mathrm{r}(5)=0.34$ & $\mathrm{r}(4)=0.92$ \\
& $\mathrm{p}=0.029$ & $\mathrm{p}=0.21$ & $\mathrm{p}=0.23$ & $\mathrm{p}=0.004$ \\
\hline
\end{tabular}

Note. Significant differences are bolded $(p<0.05)$

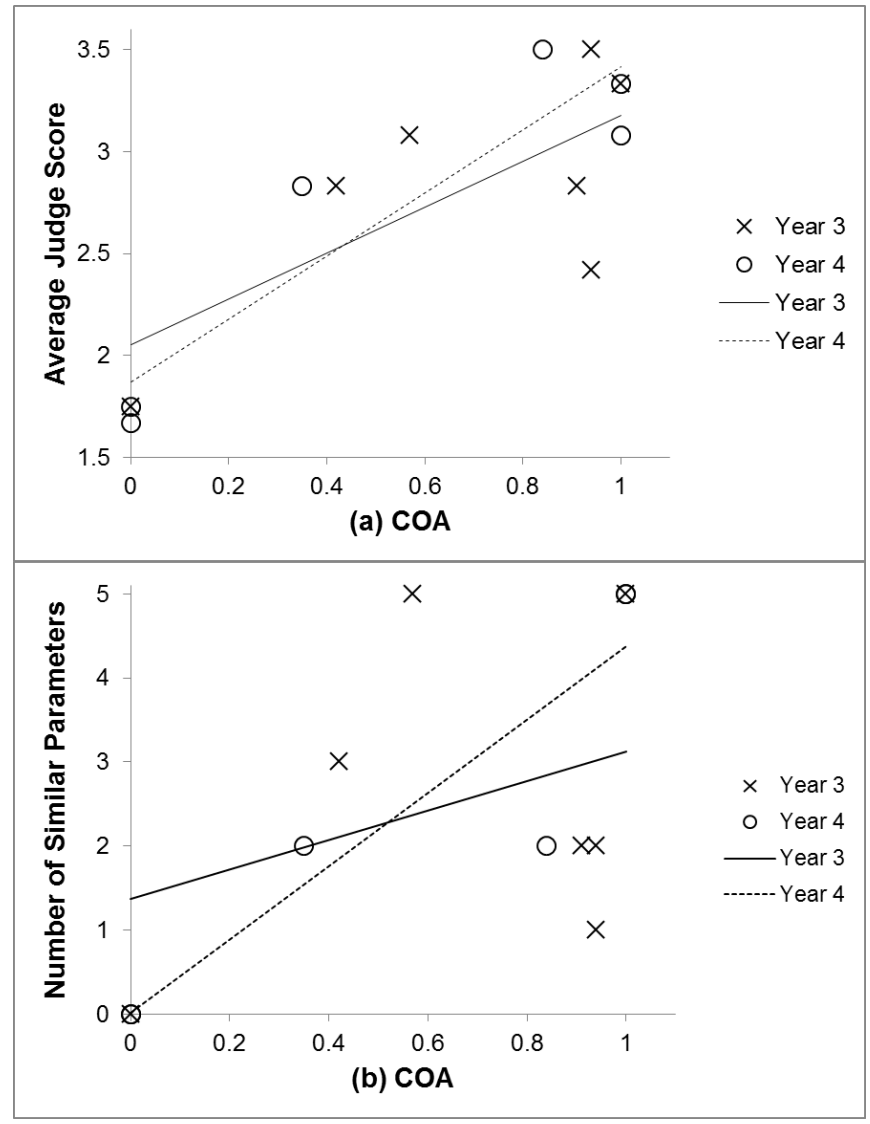

Figure 2. Relationships between COAs for mother and offspring pairs in years 3 and 4 after the offspring's birth with (a) judge scores of similarity and (b) number of similar whistle parameters (total duration, maximum frequency, minimum frequency, initial frequency, and terminal frequency). Linear regression lines are included for each year.

\section{Discussion}

The results from both judge scoring of whistle contours and the comparisons of time and frequency parameters indicate that the signature whistles of neither male nor female calves are more similar to their mothers' whistles. More female calves had whistles similar to their mothers' whistles, and a larger sample of individuals may have produced significant results. Still, our finding differs from previous research in bottlenose dolphins where male calves were more likely to have signature whistles similar to those of their mothers (Sayigh et al., 1990, 1995). 
This study is unique in that it examined the signature whistles of unrestrained, free-ranging Atlantic spotted dolphins. Studies of signature whistles have most often analyzed captive or temporarily captured bottlenose dolphins (but see Cook, Sayigh, Blum, \& Wells, 2004). Atlantic spotted dolphins have a different ecological niche, in terms of feeding strategies and diet, group size, physical size, and home range of the sexes, as compared to bottlenose dolphins (spotted: Herzing \& Johnson, 1997; bottlenose: Wells \& Scott, 1999). While both species may use signature whistles for cohesion and reunions, these ecological dissimilarities may result in different pressures for signature whistle development.

\section{Studying Whistles}

Multiple methods for measuring and comparing dolphin whistles have been utilize by researchers, and benefits and drawbacks have been discussed elsewhere (e.g., Janik, 1999). In general, each method measures only certain elements of the whistle. While whistle contour is central for individual recognition (Janik et al., 2006; Kershenbaum et al., 2013; Sayigh et al., 1998, 2005, 2007), we do not know how dolphins interpret other signals within a whistle.

We were able to compare multiple elements of a signature whistle by using complementing methods. The qualitative component of the study took advantage of complex visual pattern recognition abilities of humans, with judges comparing overall contour of the whistles - an element which is difficult to measure quantitatively. Intra-rater reliability was high, meaning each judge scored each pair generally the same in repeated comparisons. The inter-rater reliability was significant as well, but since the values for Cohen's $\kappa$ were low, it is likely that judges were focusing on different aspects of the spectrograms. The lack of repeatability between judges is a drawback to qualitative studies. Algorithm-based software programs might prove a better method for complex whistle data (Kershenbaum \& Roch, 2013; Kershenbaum et al., 2013).

Some whistles have a basic, sinusoidal structure that is similar to many other signature whistles and which may result in a high similarity rating between relatives when a whistle is simply similar to many individuals. For this reason, we compared similarity scores between an individual and a relative to the similarity scores of that individual and unrelated dolphins. Nevertheless, this method of comparison did not produce remarkably different results than a simple cut off point for similarity.

It is valuable to measure many different aspects of a vocalization, as these comparisons can give different results. Current theory predicts that contour is most important for signature information, and variations in whistles based on frequencies and duration convey additional information (Esch, Sayigh, Blum, \& Wells, 2009; Janik et al., 2006). However, even with variation within an individual, we can discriminate an individual dolphin's whistle from others, to a certain extent, based on frequency and duration information (Caldwell et al., 1990).

Partial and highly variable whistles are difficult to measure and may bias our selection and limit our studies to include only clean signals. Certain whistles, and often individuals, are excluded from study samples due to burst-pulse vocalizations in conjunction with a signature whistle (spotted: Lammers, Au, \& Herzing, 2003). A burst-pulse vocalization appears repeatedly in samples from some individuals, and may be an important feature of that individual's signature whistle. Since the frequencies of these whistles could not be accurately measured, they were excluded from our study. New techniques may be better able to analyze these types of whistles, as well as provide quantitative analysis of whistle contour (Kershenbaum et al., 2013; Kohlsdorf, Mason, Herzing, \& Starner, 2014).

\section{Home Range Differences}

In the Sarasota Bay (FL, USA) population of bottlenose dolphins, males range widely compared to females (Urian, Hofmann, Wells, \& Read, 2009). There may be an adaptive advantage for a female offspring to develop a signature whistle distinct from her mother's whistle, since she is associating frequently with her mother and her mother's associates (Sayigh et al., 1990, 1995). Atlantic spotted 
dolphins in the Bahamas occupy the same home range as their mothers for all of their lives, regardless of sex (Augliere, 2012). Consequently, both male and female Atlantic spotted dolphins associate with their mothers, her associates, and their siblings throughout their lives. Interacting individuals are expected to have distinctive signature whistles, and we expect relatives who continue to live in the same area to produce more distinctive whistles from one another. The home range differences in Atlantic spotted dolphins may explain why we did not find a sex-based difference in signature whistle similarity, as well as why judge scores in the present study were lower than those found by Sayigh et al. (1995). The ability to have a distinctive whistle that shares some similarities with a mother's whistle may act as a mechanism that allows for both individual recognition and kin recognition among matrilineal relatives.

\section{Patterns of Association}

Signature whistles are developed in a dolphin's first year, so the correlations between high $4^{\text {th }}$ year COAs and high signature whistle similarity between mother and calf are likely a reflection of a strong relationship between the pair and not the cause of the whistle similarity. Only one of the nine mother-offspring pairs (Little Gash-Little Hali) analyzed was similar in all measurements for each of the comparison methods. The COAs of Little Gash and Little Hali, measured yearly from birth to 4 years of age, were: $0.96,0.98,1.00$, and 1.00. In Atlantic spotted dolphins, COAs usually drop significantly after the first year, and again after three years (Herzing \& Brunnick, 1997). A COA of 1.0 in the fourth year demonstrates a strong relationship between Little Gash and Little Hali, which may be reflected in the high similarity of their signature whistles.

\section{Social Rank}

Sayigh et al. (1995) reported that one mother had three male calves who all developed signature whistles similar to hers, whereas another mother had three male calves who all developed whistles unlike hers. Our study included two mothers with more than one of their offspring (Dos and Luna). Of Dos's three calves, one produced signature whistles that were similar to hers in both qualitative and quantitative measures, another had no similarities, and one was similar for some of the measured parameters. Both of Luna's calves produced signature whistles that were similar to her signature whistle with the qualitative measure, but not with quantitative measures.

A mother's social rank may influence the degree of similarity between mother and offspring signature whistles. Dominance, or status, within fission-fusion communities is very complex. For example, only after careful analysis of 35 years of data was a dominance hierarchy apparent in female chimpanzees (Pan troglodytes: Pusey, Williams, \& Goodall, 1997). Once the rankings of female chimpanzees were determined, unusual dispersal differences observed in offspring were better understood. We may observe a similar occurrence in dolphin offspring where offspring of dominant mothers benefit by advertising their maternal relationship (Sayigh et al., 1990, 1995).

Studying the similarities and differences in signature whistles between mothers and offspring may help illuminate the mechanisms used in the development of a signature whistle and also the ultimate basis for signature whistle use in a dolphin community. Continued research on the development and use of dolphin vocalizations will help us to understand communication, cognition, and social behavior in these complex organisms.

\section{Acknowledgements}

This project was funded by the Wild Dolphin Project and Florida Atlantic University and conducted under a research permit from the Bahamian Department of Fisheries. Special thanks to Courtney E. Bender, Lillian S. Welsh, Kyle A. Bartow, Cindy R. Elliser, Blake C. Jones, David F. Bjorklund, and W. Randy Brooks. Thank you to the crew, volunteers, and members of the Wild Dolphin Project. We also thank Arik Kershenbaum and two anonymous referees for their helpful comments. 
Correspondence regarding this article should be addressed to Sara E. Bebus, Department of Biological Sciences, University of Memphis, 3774 Walker Ave., Memphis, TN, 38152. (sarabebus@ gmail.com).

\section{References}

Augliere, B. (2012). Home on the range: spatial ecology of Atlantic spotted dolphins (Stenella frontalis) in the Bahamas (Unpublished master's thesis). Florida Atlantic University, Boca Raton, FL.

Burris, J. (2004). Signature whistle stability in wild female Atlantic spotted dolphins, Stenella frontalis (Unpublished master's thesis). Florida Atlantic University, Boca Raton, FL.

Cairns, S. J., \& Schwager, S. J. (1987). A comparison of association indices. Animal Behaviour, 35, 1454-1469. doi: 10.1016/S0003-3472(87)80018-0

Caldwell, M. C., \& Caldwell, D. K. (1965). Individual whistle contours in bottlenosed dolphins (Tursiops truncatus). Nature, 207, 434-435. doi: 10.1038/207434a0

Caldwell, D. K., \& Caldwell, M. C. (1966). Observations on the distribution, coloration, behavior and audible sound production of the spotted dolphin, Stenella plagiodon. Contributions in Science, 104, 1-28.

Caldwell, M. C., \& Caldwell, D. K. (1968). Vocalization of native captive dolphins in small groups. Science, 159, 1121-1123. doi: $10.1126 /$ science.159.3819.1121

Caldwell, M. C., \& Caldwell, D. K. (1971). Statistical evidence for individual signature whistles in Pacific whitesided dolphins, Lagenorhynchus obliquidens. Cetology, 3, 1-9.

Caldwell, M. C., \& Caldwell, D. K. (1979). The whistle of the Atlantic bottlenosed dolphin - ontogeny. In H. E. Winn \& B. L. Oll (Eds.), Behavior of marine animals, Vol. 3. (pp. 369-401). New York: Plenum Press.

Caldwell, D. K., Caldwell, M. C., \& Miller, J. F. (1969). Three brief narrow-band sound emissions by a captive male Risso's dolphin, Grampus griseus. Southern California Academy of Sciences Bulletin, 68, 252-256.

Caldwell, M. C., Caldwell, D. K., \& Miller, J. F. (1973). Statistical evidence for individual signature whistles in the spotted dolphin, Stenella plagiodon. Cetology, 16, 1-21.

Caldwell, M. C., Caldwell, D. K., \& Tyack, P. L. (1990). A review of the signature whistle hypothesis for the Atlantic bottlenose dolphin, Tursiops truncatus. In S. Leatherwood \& R. Reeves (Eds.), The bottlenose dolphin: Recent progress in research (pp. 199-234). San Diego: Academic Press.

Cook, M. L., Sayigh, L. S., Blum, J. E., \& Wells, R. S. (2004). Signature-whistle production in undisturbed freeranging bottlenose dolphins (Tursiops truncatus). Proceedings of the Royal Society B, 271, 1043-1050. doi: 10.1098/rspb.2003.2610

de Figueiredo, L. D., \& Simão, S. M. (2009). Possible occurrence of signature whistles in a population of Sotalia guianensis (Cetacea, Delphinidae) living in Sepetiba Bay, Brazil. Journal of the Acoustical Society of America, 126, 1563-1569. doi: 10.1121/1.3158822

Elliser, C. R., \& Herzing, D. L. (2012). Community structure and cluster definition of Atlantic spotted dolphins, Stenella frontalis, in the Bahamas. Marine Mammal Science, 28, e486-e502. doi: 10.1111/j.17487692.2012.00576.x

Elliser, C. R. \& Herzing, D. L. (2014). Long-term social structure of a resident community of Atlantic spotted dolphins, Stenella frontalis, in the Bahamas 1991-2002. Marine Mammal Science, 30, 308-328. doi: $10.1111 / \mathrm{mms} .12039$

Esch, H. C., Sayigh, L. S., Blum, J. E., \& Wells, R. S. (2009). Whistles as potential indicators of stress in bottlenose dolphins (Tursiops truncatus). Journal of Mammalogy, 90, 638-650. doi: 10.1644/08-MAMM-A-069R.1

Esch, H. C., Sayigh, L. S., \& Wells, R. S. (2009). Quantifying parameters of bottlenose dolphins signature whistles. Marine Mammal Science, 25, 976-986. doi: 10.1111/j.1748-7692.2009.00289.x

Fripp, D. (2005). Bubblestream whistles are not representative of a bottlenose dolphin's vocal repertoire. Marine Mammal Science, 21, 29-44. doi: 10.1111/j.1748-7692.2005.tb01206.x

Fripp, D., Owen, C., Quintana-Rizzo, E., Shapiro, A., Buckstaff, K., Jankowski, K., ...Tyack, P. (2005). Bottlenose dolphin (Tursiops truncatus) calves appear to model their signature whistles on the signature whistles of community members. Animal Cognition, 8, 17-26. doi: 10.1007/s10071-004-0225-z

Gridley, T., Cockcroft, V. G., Hawkins, E. R., Blewitt, M. L., Morisaka, T., \& Janik, V. M. (2014). Signature whistles in free-ranging populations of Indo-Pacific bottlenose dolphins, Tursiops aduncus. Marine Mammal Science, 30, 512-527. doi: 10.1111/mms.12054

Harley, H. E. (2008). Whistle discrimination and categorization by the Atlantic bottlenose dolphin (Tursiops truncatus): A review of the signature whistle framework and a perceptual test. Behavioural processes, 77, 243-268. doi: 10.1016/j.beproc.2007.11.002 
Herzing, D. L. (1996). Vocalizations and associated underwater behavior of free-ranging Atlantic spotted dolphins and bottlenose dolphins. Aquatic Mammals, 22, 61-79.

Herzing, D. L. (1997). The life history of free-ranging Atlantic spotted dolphins: age classes, color phases, and female reproduction. Marine Mammal Science, 13, 576-595. doi: 10.1111/j.1748-7692.1997.tb00085.x

Herzing, D. L., \& Brunnick, B. J. (1997). Coefficients of association of reproductively active female Atlantic spotted dolphins, Stenella frontalis. Aquatic Mammals, 23, 155-162.

Herzing, D. L., Deecke, V., \& Brunnick, B. (1999). Signature whistles of Atlantic spotted dolphins (Stenella frontalis): Measures of similarity and biological significance. Proceedings of the Thirteenth Biennial Conference on the Biology of Marine Mammals. Maui, HI.

Herzing, D. L., \& Johnson, C. M. (1997). Interspecific interactions between Atlantic spotted dolphins (Stenella frontalis) and bottlenose dolphins (Tursiops truncatus) in the Bahamas, 1985-1995. Aquatic Mammals, 23, 85-99.

Janik, V. M. (1999). Pitfalls in the categorization of behaviour: A comparison of dolphin whistle classification methods. Animal Behaviour, 57, 133-143. doi: 10.1006/anbe.1998.0923

Janik, V. M. (2000). Whistle matching in wild bottlenose dolphins (Tursiops truncatus). Science, 289, 1355-1357. doi: $10.1126 /$ science.289.5483.1355

Janik, V. M., \& Sayigh, L. S. (2013). Communication in bottlenose dolphins: 50 years of signature whistle research. Journal of Comparative Physiology A, 199, 479-489. doi: 10.1007/s00359-013-0817-7

Janik, V. M., Sayigh, L. S., \& Wells, R. S. (2006). Signature whistle shape conveys identity information to bottlenose dolphins. Proceedings of the National Academy of Sciences, 103, 8239-8297. doi: 10.1073/pnas.0509918103

Janik, V. M., \& Slater, P. J. B. (1997). Vocal learning in mammals. Advances in the Study of Behavior, 26, 59-99. doi: 10.1016/S0065-3454(08)60377-0

Janik, V. M., \& Slater, P. J. B. (1998). Context-specific use suggests that bottlenose dolphin signature whistles are cohesion calls. Animal Behaviour, 56, 829-838. doi: 10.1006/anbe.1998.0881

Kershenbaum, A., \& Roch, M. A. (2013). An image processing based paradigm for the extraction of tonal sounds in cetacean communications. Journal of the Acoustical Society of America, 134, 4435-4445. doi: $10.1121 / 1.4828821$

Kershenbaum, A., Sayigh, L. S., \& Janik, V. M. (2013). The encoding of individual identity in dolphin signature whistles: How much information is needed? PloS one, 8, e77671. doi: 10.1371/journal.pone.0077671

King, S. L., \& Janik, V. M. (2013). Bottlenose dolphins can use learned vocal labels to address each other. Proceeding of the National Academy of Sciences, 110, 13216-13221. doi: 10.1073/pnas.1304459110

King, S. L., Sayigh, L. S., Wells, R. S., Fellner, W., \& Janik, V. M. (2013). Vocal copying of individually distinctive signature whistles in bottlenose dolphins. Proceedings of the Royal Society B, 280, 20130053. doi: $10.1098 / \mathrm{rspb} .2013 .0053$

Kohlsdorf, D., Mason, C., Herzing, D., \& Starner, T. (2014). Probabilistic extraction and discovery of fundamental units in dolphin whistles. Proceedings of the Thirty-ninth International Conference on Acoustics, Speech, and Signal Processing. Florence, Italy. doi: 10.1109/ICASSP.2014.6855208

Lammers, M. O., Au, W. W., \& Herzing, D. L. (2003). The broadband social acoustic signaling behavior of spinner and spotted dolphins. Journal of the Acoustical Society of America, 114, 1629-1639. doi: $10.1121 / 1.1596173$

McCowan, B., \& Reiss, D. (1995a). Quantitative comparison of whistle repertoires from captive adult bottlenose dolphins (Delphinidae, Tursiops truncatus): A re-evaluation of the signature whistle hypothesis. Ethology, 100, 194-209. doi: 10.1111/j.1439-0310.1995.tb00325.x

McCowan, B., \& Reiss, D. (1995b). Whistle contour development in captive-born infant bottlenose dolphins (Tursiops truncatus): Role of learning. Journal of Comparative Psychology, 109, 242-260. doi: 10.1037/0735-7036.109.3.242

Miksis, J. L., Tyack, P. L., \& Buck, J. R. (2002). Captive dolphins, Tursiops truncatus, develop signature whistles that match acoustic features of human-made model sounds. Journal of the Acoustical Society of America, 112, 728-739. doi: 10.1121/1.1496079

Pusey, A. E., Williams, J., \& Goodall, J. (1997). The influence of dominance rank on the reproductive success of female chimpanzees. Science, 277, 828-831. doi: 10.1126/science.277.5327.828

Quick, N. J., \& Janik, V. M. (2012). Bottlenose dolphins exchange signature whistles when meeting at sea. Proceedings of the Royal Society B, 279, 2539-2545. doi: 10.1098/rspb.2011.2537

Reiss, D. (1988). Observations on the development of echolocation in young bottlenose dolphins. In P. E. Nachtigall \& P. W. B. Moore (Eds.), Animal sonar (pp. 121-127). New York: Plenum Publishing. 
Sayigh, L. S., Esch, H. C., Wells, R. S., \& Janik, V. M. (2007). Facts about signature whistles of bottlenose dolphins, Tursiops truncatus. Animal Behaviour, 74, 1631-1642. doi: 10.1016/j.anbehav.2007.02.018

Sayigh, L. S., Janik, V. M., \& Wells, R. S. (2005). What's in a voice? Cues used by dolphins in individual recognition of signature whistles. Journal of the Acoustical Society of America, 118, 1906-1906. doi: $10.1121 / 1.4780125$

Sayigh, L. S., Tyack, P. L., Wells, R. S., \& Scott, M. D. (1990). Signature whistles of free-ranging bottlenose dolphins: Stability and mother-offspring comparisons. Behavioral Ecology and Sociobiology, 26, 247-260. doi: 10.1007/BF00178318

Sayigh, L. S., Tyack, P. L., Wells, R. S., Scott, M. D., \& Irvine, A. B. (1995). Sex differences in signature whistle production of free-ranging bottlenose dolphins. Behavioral Ecology and Sociobiology, 36, 171-177. doi: 10.1007/BF00178318

Sayigh, L. S., Tyack, P. L., Wells, R. S., Solow, A. R., Scott, M. D., \& Irvine, A. B. (1998). Individual recognition in wild bottlenose dolphins: A field test using playback experiments. Animal Behaviour, 57, 41-50. doi:10.1006/anbe.1998.0961

Smolker, R. A., Mann, J., \& Smuts, B. B. (1993). Use of signature whistles during separations and reunions by wild bottlenose dolphin mothers and infants. Behavioral Ecology and Sociobiology, 33, 393-402. doi: 10.1007/BF00170254

Smolker, R., \& Pepper, J. W. (1999). Whistle convergence among allied male bottlenose dolphins (Delphinidae, Tursiops sp.). Ethology, 105, 595-617. doi: 10.1046/j.1439-0310.1999.00441.x

Tyack, P. L. (1997). Development and social functions of signature whistles in bottlenose dolphins, Tursiops truncatus. Bioacoustics, 8, 21-46. doi: 10.1080/09524622.1997.9753352

Tyack, P. L., \& Sayigh, L. S. (1997). Vocal learning in cetaceans. In C. Snowden \& M. Hausberger (Eds.), Social influences on vocal development (pp. 208-233). Cambridge, UK: Cambridge University Press.

Urian, K. W., Hofmann, S., Wells, R. S., \& Read, A. J. (2009). Fine-scale population structure of bottlenose dolphins (Tursiops truncatus) in Tampa Bay, Florida. Marine Mammal Science, 25, 619-638. doi: 10.1111/j.1748-7692.2009.00284.x

Van Parijs, S. M., \& Corkeron, P. J. (2001). Evidence for signature whistle production by a Pacific humpback dolphin, Sousa chinensis. Marine Mammal Science, 17, 944-949. doi: 10.1111/j.1748-7692.2001.tb01308.x

Watwood, S. L., Tyack, P. L., \& Wells, R. S. (2004). Whistle sharing in paired bottlenose dolphins, Tursiops truncatus. Behavioral Ecology and Sociobiology, 55, 531-543. doi: 10.1007/s00265-003-0724-y

Wells, R. S. \& Scott, M. D. (1999). Bottlenose dolphin, Tursiops truncatus (Montagu, 1821). In S. H. Ridgway \& R. Harrison (Eds.), Handbook of marine mammals, Vol. 6 (pp. 137-182). San Diego: Academic Press.

Welsh, L. S., \& Herzing, D. L. (2008). Preferential association among kin exhibited in a population of Atlantic spotted dolphins (Stenella frontalis). International Journal of Comparative Psychology, 21, 1-11. 